\title{
IMPLEMENTATION OF A GENERAL REAL-TIME VISUAL ANOMALY DETECTION SYSTEM VIA SOFT COMPUTING
}

\author{
Jesús A. Dominguez, Dynacs Inc., AAAI member \\ Steve Klinko, Dynacs Inc., IEEE member \\ Bob Ferrell, NASA, AAAI member \\ Kennedy Space Center, Florida USA. \\ The $10^{\text {th }}$ IEEE International Conference on Fuzzy Systems \\ The University of Melbourne, Australia \\ December 2-5, 2001
}

T1: Pattern Recognition and Image Processing

Keywords: soft computing, vision computing, visual anomaly detection, fuzzy reasoning, digital image analysis, visual learning machine.

Contact Author: Jesús A. Dominguez, jesus.dominguez-1@ksc.nasa.gov 321/867-6696, 321/867-6500 (fax), Kennedy Space Center

EDL Building, Room 230G, Kennedy Space Center, Florida 32815

\begin{abstract}
The intelligent visual system detects anomalies or defects in real time under normal lighting operating conditions. The application is basically a learning machine that integrates fuzzy logic (FL), artificial neural network (ANN), and generic algorithm (GA) schemes to process the image, run the learning process, and finally detect the anomalies or defects. The system acquires the image, performs segmentation to separate the object being tested from the background, preprocesses the image using fuzzy reasoning, performs the final segmentation using fuzzy reasoning techniques to retrieve regions with potential anomalies or defects, and finally retrieves them using a learning model built via $\mathrm{ANN}$ and $\mathrm{GA}$ techniques.

FL provides a powerful framework for knowledge representation and overcomes uncertainty and vagueness typically found in image analysis. ANN provides learning capabilities, and GA leads to robust learning results. An application prototype currently runs on a regular PC under Windows NT, and preliminary work has been performed to build an embedded version with multiple image processors.

The application prototype is being tested at the Kennedy Space Center (KSC), Florida, to visually detect anomalies along slide basket cables utilized by the astronauts to evacuate the NASA Shuttle launch pad in an emergency. The potential applications of this anomaly detection system in an open environment are quite wide. Another current, potentially viable application at NASA is in detecting anomalies of the NASA Space Shuttle Orbiter's radiator panels.
\end{abstract}




\section{INTRODUCTION}

Automated real-time visual inspection under normal lighting is a very complex task. The main problems (which include image vagueness due to lack of proper lighting, extremely high data flow, noise influence, interclass similarity and interclass diversity, large number of defect classes, dynamic defect population, and economical justification) are not solved yet.

Handling uncertainty on image features (image enhancement, edginess grading, background extraction, noise elimination, etc.) and performing visual classification in real time are the main problem characteristics of a visual anomaly detection system. Computer vision, in general, and particularly anomaly detection under normal lighting conditions are very difficult problems. This is in part because of the many levels of uncertainty inserted into the data by a variety of processes from the original sensor through several levels of interpretation.

Mainstream approaches to dealing with this uncertainty include the use of thresholds, which are difficult to choose and cause information loss, and probabilistic methods, which rely on knowledge about prior distribution that is often not available. Other approaches are based on a morphological filtering method and neural-network classifier, but they face problems performing image threshold and feature extraction for some specific anomalies or defects under normal lighting conditions. Integrating the three constituents of soft computing - FL, AN, and GA - solves these problems.

Image analysis leading to either detection of anomalies or performance of pattern recognition is not only an "inverse inspection problem" without a single mathematical solution, but is also complicated by external sources of uncertainty such as the conditions of the image formation and the final decision-making approach. Thus, the need for dealing with uncertainty in computer vision is well accepted. However, most of the work in this area has used fixed thresholds or probabilities approaches. Although fuzzy systems are basically out of the mainstream of computer vision, they pose great value for addressing uncertainty issues that are not adequately dealt with by current methods.

\section{SYSTEM DESCRIPTION}

The system acquires the images from a video camera via either an analog or digital image grabber. Using fuzzy reasoning, the image undergoes preprocessing that includes primary segmentation, object extraction, enhancement, filtering, and edginess detection. An adaptive fuzzy threshold binaries the preprocessed image, and a selective blob detector picks image regions with high edginess activity. The system computes morphological, geometrical, and fuzzy edginess grading values for each region and uses them as inputs of the learning feature via GA-ANN schemes. A schematic of the system is shown in figure 1 .

The system has several innovative features to perform image analysis based on fuzzy reasoning and integrates them with a learning machine built using ANN and GA schemes. The image analysis needed to perform enhancement, filtering, edginess grading, and segmentation is based on a unique and innovative fuzzy reasoning approach. The application is basically a general-purpose and realtime anomaly detection system that does not require special lighting and can be embedded to a portable application in an open environment. 


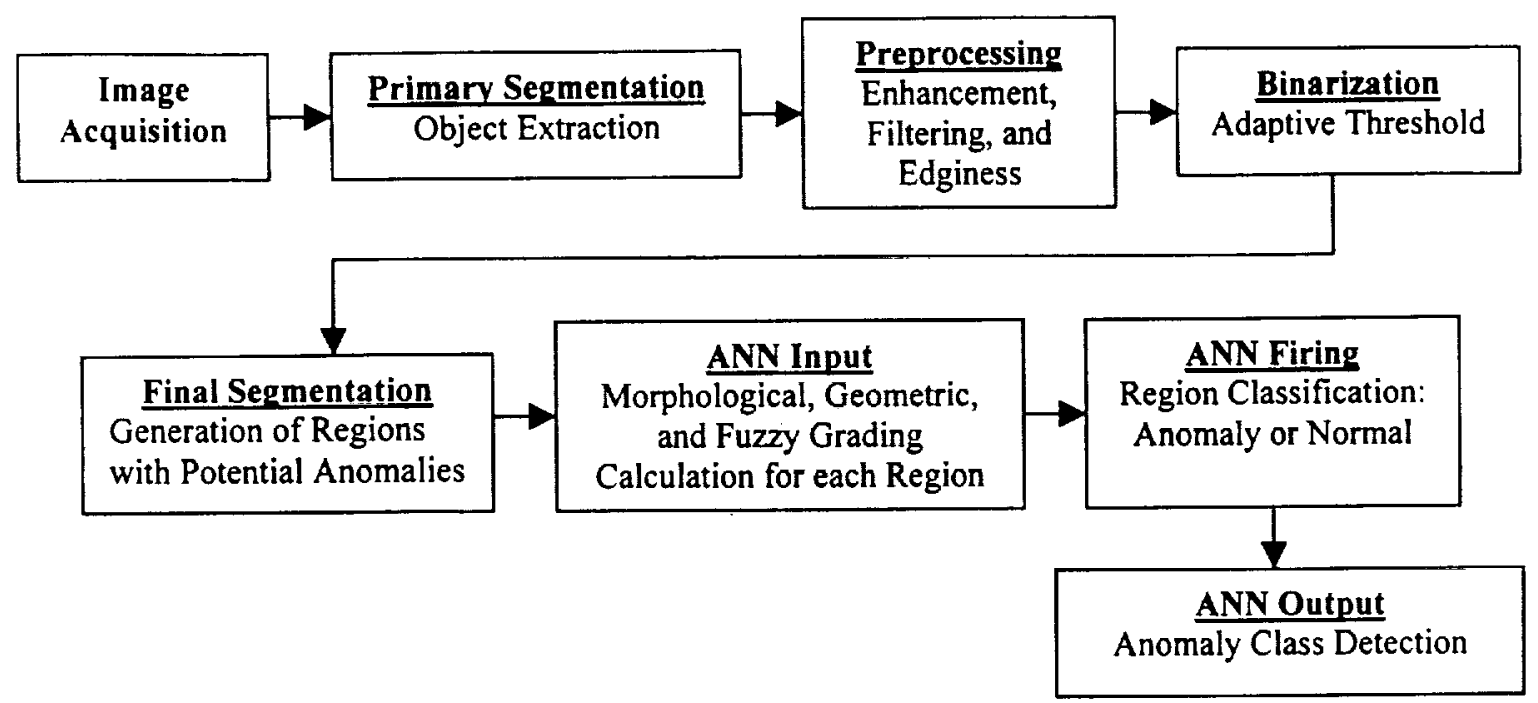

Figure 1. Schematic of the intelligent visual anomaly detection system.

An innovative concept of edginess grading based on fuzzy reasoning implemented here makes it possible to identify in real time potential anomaly spots on the images. This approach quantifies the degree of edginess and uses its value as an input for the learning scheme via ANN and GA. The learning scheme also computes several morphological and geometrical features of these spots and uses them as inputs.

Visual analysis, in general, requires a large amount of CPU power leading to the degradation of its real-time performance. Anomaly detection systems have to be run under a "hard" real-time constraint. The code of all image operators based on fuzzy reasoning and used to implement the image preprocessing stages (segmentation, enhancement, filtering, edginess grading, and binarization) is optimized to minimize execution overhead and keep the system running under "hard" real-time performance requirements.

The system may run on either a regular PC under Windows NT or an embedded version for portability. Hardware required to build the system includes a video camera, an image grabber, an image processor (a PC processor or a stand-alone image processor in the embedded application), and the respective peripheral devices (monitor, mouse, keyboard, and data storage medium) attached to the image processor. The software development tools used in the implementation of this system include Microsoft Visual C++, Microsoft Foundation Class (MFC), and two, commercial software packages: Wit image analysis software from Logical Vision and NeuroShell Classifier from Ward Systems.

Wit software allows the developer to build customized image operators in $\mathrm{C}$ and a front end to make the application friendly and transparent to the end user. We designed and built most of the image operators, including those needed to perform image preprocessing, via fuzzy reasoning. The ANN scheme is based on Probabilistic Neural Nets (PNN) but is formulated a little differently, based on $\mathrm{GA}$ as opposed to a network structure. 
PNN's instantly train, unlike back propagation. In fact, they do not really train at all; they memorize, and the only freedom they have to generalize is to vary the smoothing factors. So the approach used in NeuroShell memorizes all of the patterns in the training set. Then it begins generating genetic individuals with GA. Each individual is a set of smoothing factors, which, when applied to PNN, makes a complete net. That net is tested against the test set, and the results become the GA fitness function. The GA generates the smoothing factors that perform the best against the test set, but all nets thus generated by the GA are trained on the training set (memorized). NeuroShell Classifier contains this new GA-PNN network paradigm. NeuroShell Classifier is spawned via DLL functions to perform training and firing of the GA-PNN classification net.

On the end-user side, all the features involved in the system are completely transparent. The end software product offers a friendly and easy-to-use template (all built via MFC) with a menu to select and perform training, analysis, and detection. The system has two end products - the development software package that includes all the system features and the run-time package designed to just run the anomaly detection system in the field.

\section{FUZZY REASONING AND ANN INTEGRATION}

Visual inspection faces many levels of uncertainty inserted into the data by a variety of processes from the original sensor through several levels of interpretation. The integration of the three constituents of soft computing - FL, ANN, and GA - is used to manage these levels of uncertainty. Soft computing differs from conventional (hard) computing in that, unlike hard computing, it is tolerant of imprecision, uncertainty, and partial truth. In effect, the role model for soft computing is the human mind.

Fuzzy reasoning and ANN lead to a symbiotic relationship in which fuzzy systems provide a powerful framework for knowledge representation, while ANN provides learning capabilities and exceptional suitability for computationally efficient hardware implementation. Generating optimal smoothing factors via GA approach significantly enhances the performance and robustness of the PNN.

The anomaly detection system uses a fuzzy reasoning approach to perform image preprocessing, adaptive image binarization, and image edginess grading. The fuzzy reasoning leads to the identification of image regions with important edginess activity. An additional system module retrieves those regions; computes morphological, geometric, and fuzzy edginess grading values for each region; and uses them to perform training and generate the respective ANN model.

\section{PROTOTYPE TEST}

NASA has built an inspection mechanism called Cable and Line Inspection Mechanism (CLIM) to provide a means of automated image acquisition of the fourteen 1000 -foot slidewires used in the emergency egress system for the NASA Space Shuttle at KSC. CLIM eliminates the hazardous, manpower-intensive, and time-consuming methods previously required to manually maintain the emergency egress system at peak performance. The inspection of the emergency egress system using CLIM still requires having a person continuously watch cable images for more than 48 hours. The intelligent anomaly detection system will be integrated to CLIM to completely eliminate the need for human intervention in detecting anomalies; the system will not only detect the anomaly, but 
also document it. Figure 2 shows a picture of CLIM climbing one of the fourteen 1000-foot slidewires used in the emergency egress system for the NASA Space Shuttle at KSC.

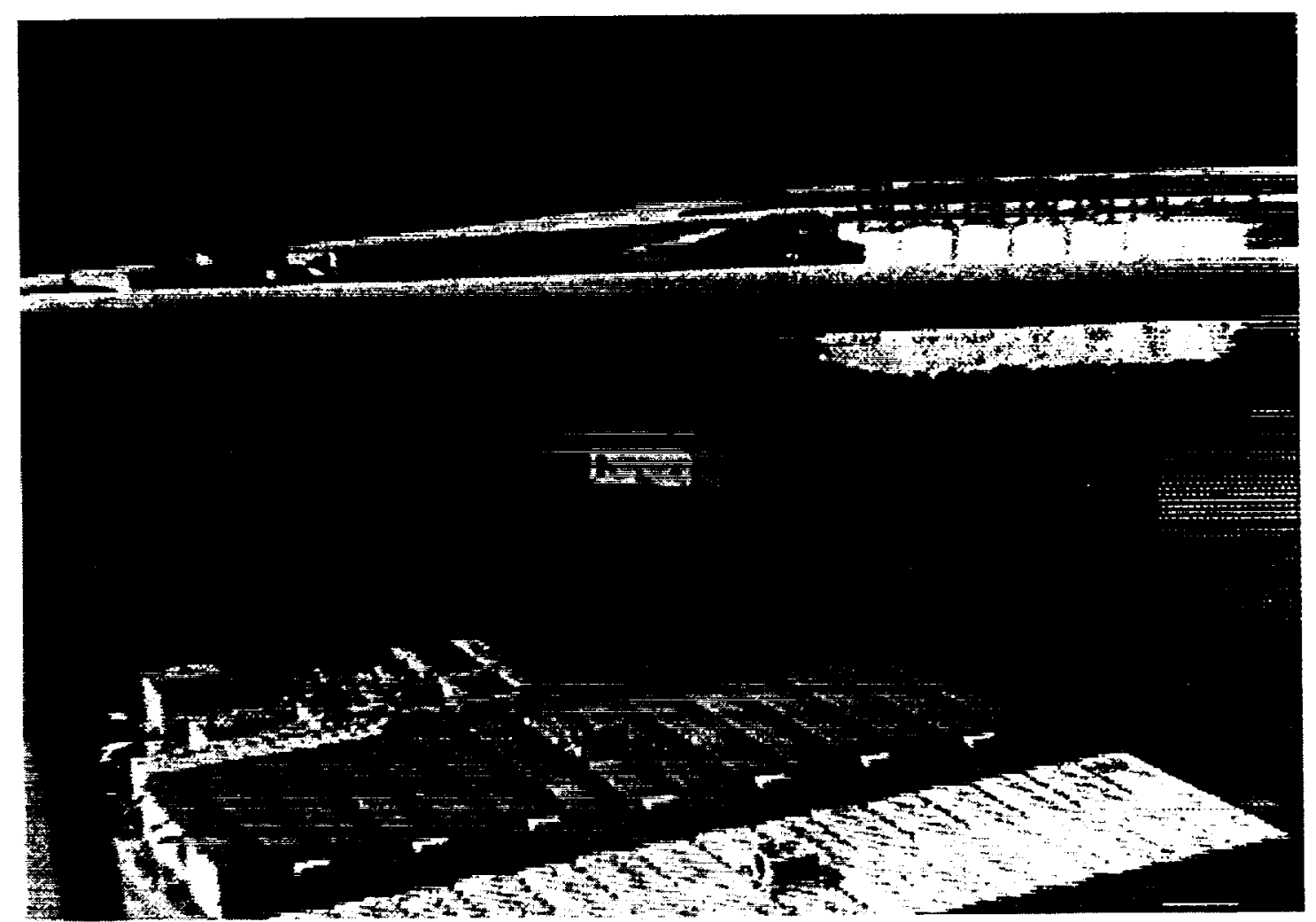

Figure 2. CLIM being tested at one of the slidewires used in the emergency egress system for the Space Shuttle at the Kennedy Space Center. Images acquired by CLIM are being used to test the intelligent real-time anomaly detection system.

Images acquired by CLIM are being used to test the intelligent real-time anomaly detection system. Figures 3.1 through 3.5 show a real-time sequence of image events performed by the detection system: image acquired by CLIM (figure 3.1), cable image extracted from the background and preprocessed via fuzzy reasoning (figure 3.2), image binarization via adaptive fuzzy threshold (figure 3.3), identification of regions with potential anomalies (figure 3.4), and final anomaly detection (figure 3.5 ).

The anomaly shown in figure 3.1 was one of four other similar anomaly occurrences rated under the same type or class to test the application. This anomaly classification identifies small strand pieces slightly separated from the cable main strands. All five anomalies were located in 40 feet of cable, and all five anomalies were repeatedly detected by the system. An average of 4 false anomalies were detected in all runs ( 40 feet each run). Considering the number of points used for training (only five for anomaly and more than 400 for normal), the system performance supersedes the original expectation. The true-true detection rate was 100 percent and the true-false rate was lower than 0.02 percent. Further tests using different anomaly and normal classifications are currently being planned. 


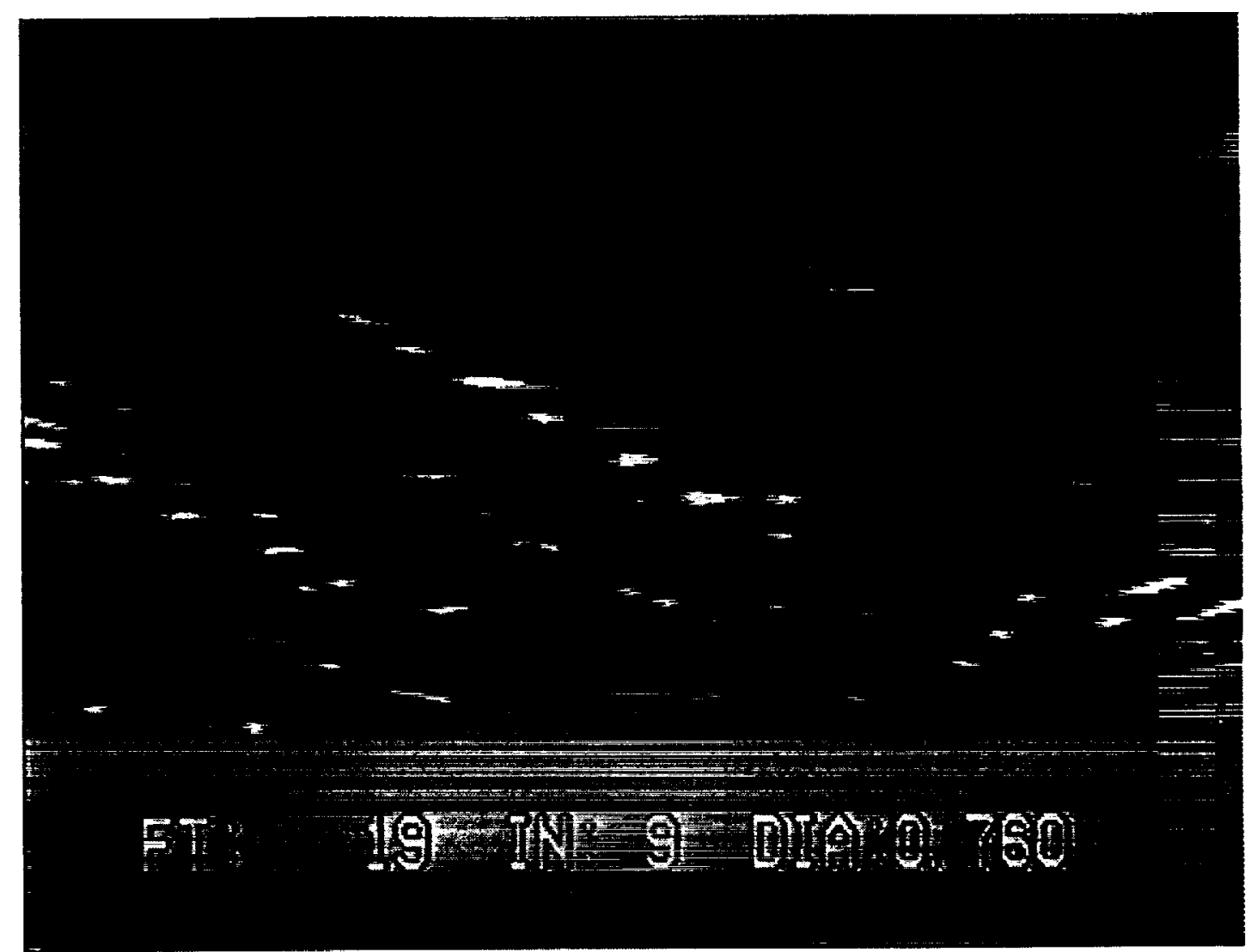

Figure 3.1. Image acquired by CLIM holding a mild anomaly.

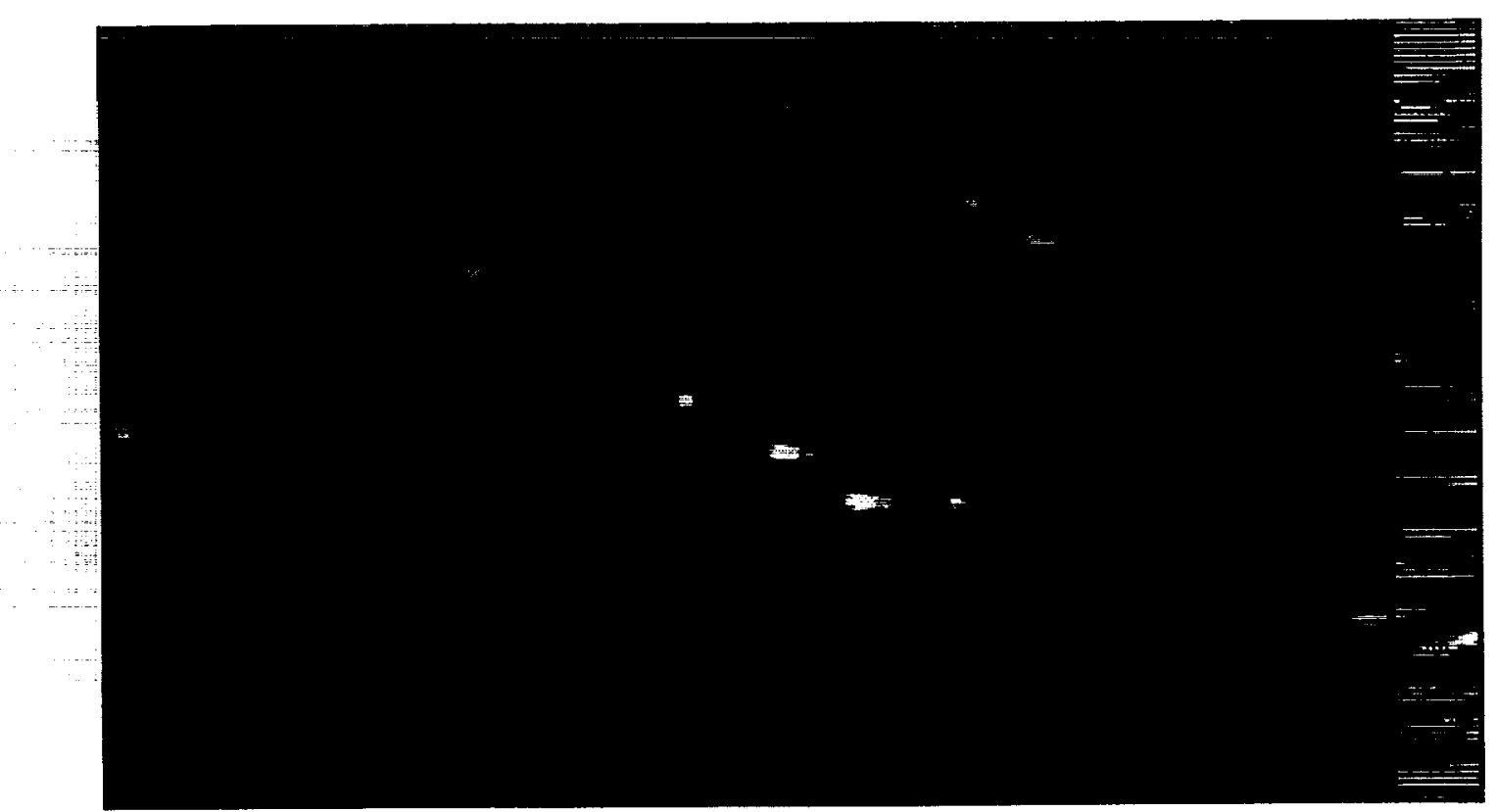

Figure 3.2. Extracted and preprocessed image based on fuzzy reasoning. 


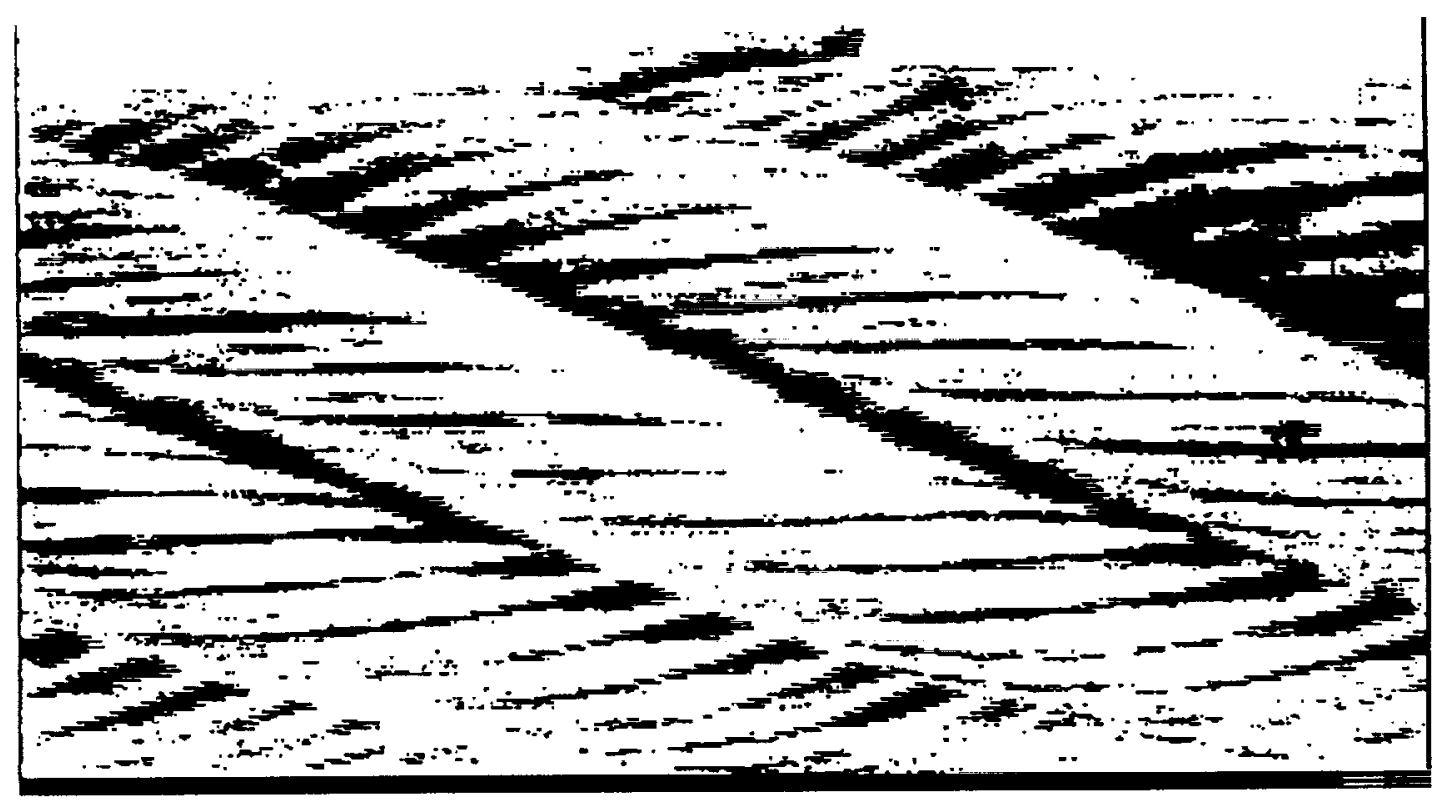

Figure 3.3. Adaptive fuzzy image threshold and binary image generation.

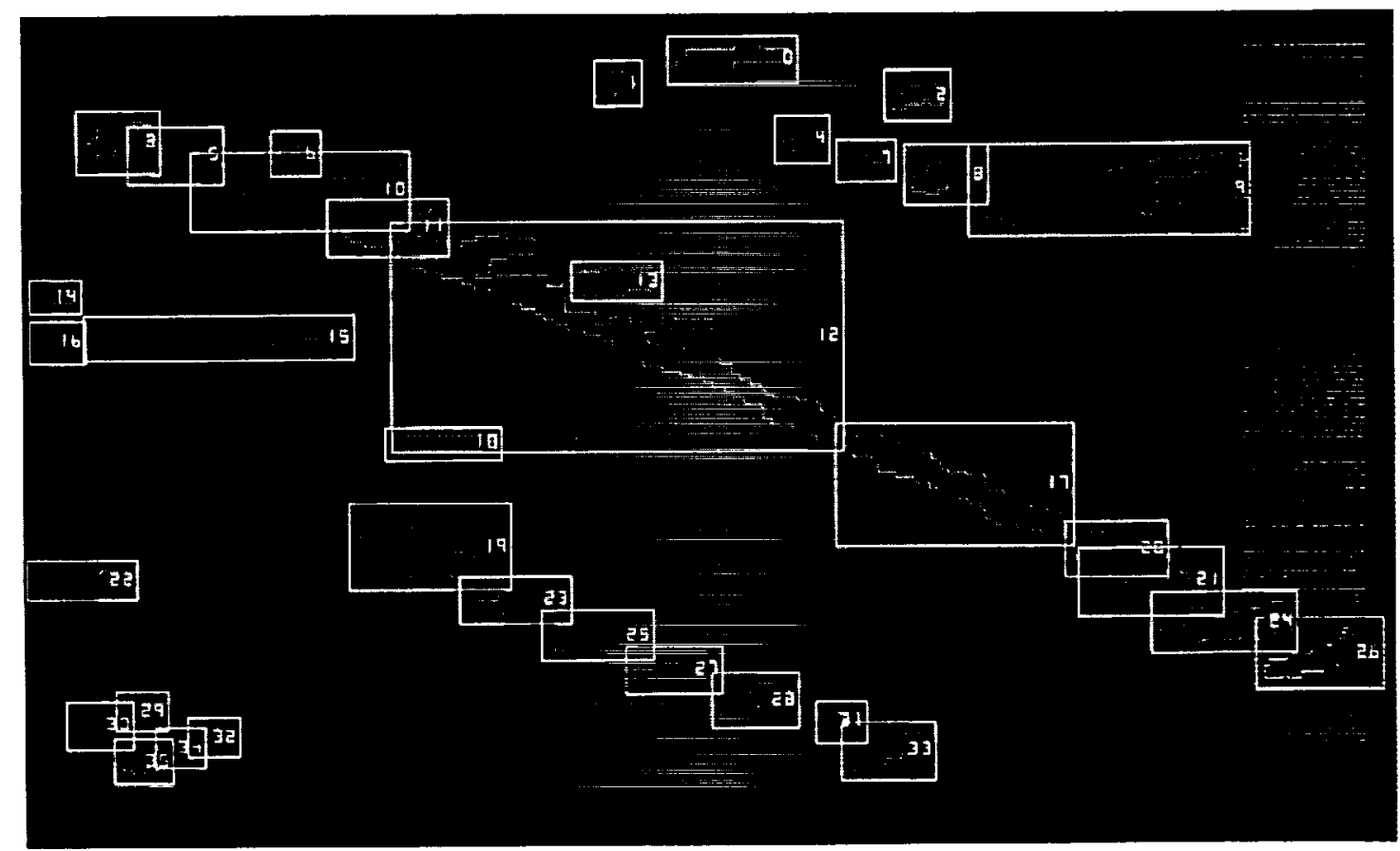

Figure 3.4. Image skeleton indicating perimeter of individual regions with potential anomalies. Region 0 contains an actual mild anomaly (see figure 3.1). 


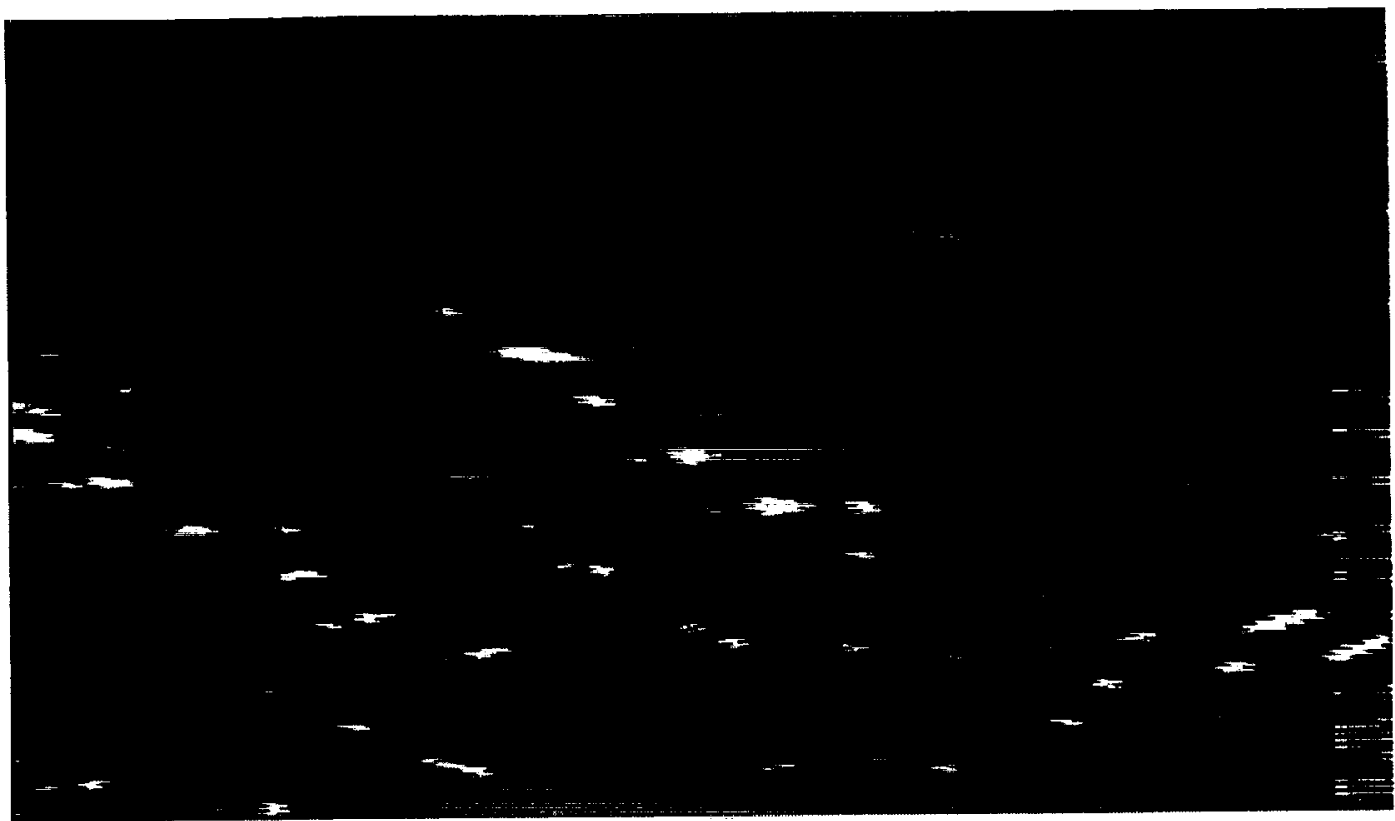

Figure 3.5. Mild anomaly detection in real time indicated by a rectangle corresponding to region 0 .

\section{CONCLUSIONS}

The CLIM project, which has been designed to visually document the slide basket cables utilized by the astronauts to evacuate the NASA Shuttle launch pad in an emergency, is being used to test the system. Preliminary tests, using images recorded from a slide basket cable mounted in the laboratory, show promising results.

The potential applications of this anomaly detection system in an open environment are quite wide because there is no need for special lighting. One immediate application at KSC is the detection of anomalies of the NASA Space Shuttle Orbiter's radiator panels. Potential applications include automated visual inspection on spacecraft, ground, and flight equipment. The embedded portable version of this system makes the visual inspection of this type of equipment (including spacecraft fuselage) quite feasible in an open environment.

The fuzzy operators built to preprocess the image can be incorporated into other computer vision systems. Current visual systems that rely on clustering, regardless of the space in which clustering occurs, and the visual systems that are based on thresholds can be generalized to use fuzzy thresholding to delay commitment. Finally, probabilistic visual systems that rely on prior distribution that is not really available can be recast as systems that match input data to fuzzy models. 


\section{REFERENCES}

Caudill, M., and C. Butler, Naturally Intelligent Systems, The MIT Press, Cambridge, Massachusetts, 1992.

Chen, C.H., Fuzzy Logic and Neural Network Handbook, McGraw-Hill (Series on Computer Engineering), New York, 1992.

Chi, Z., H. Yan, and T. Pham, "Fuzzy Algorithms: With Applications to Images Processing and Pattern Recognition," Advances in Fuzzy Systems - Applications and Theory, Vol. 10, World Scientific Publishing Co., Pte. Ltd., Singapore.

Cox, E., Fuzzy Systems Handbook, 2d ed., Academic Press, AP Professional, 1998.

Jain, A.K., Fundamentals of Image Processing, Prentice-Hall, Englewood Cliffs, New Jersey, 1998.

Laplante, P.A., and A.D. Stoyenko, Real-Time Imaging: Theory, Techniques, and Applications, IEEE Press, 1996.

Lie, W.N., "Automatic Target Segmentation by Locally Adaptive Image Thresholding," IEEE Trans. Image Processing, Vol. 4, July 1995, pp. 1036-1041.

Lippmann, R.P., and B. Gold, "Neural-Net Classifier Useful for Speech Recognition," IEEE International Conference - Neural Networks, Vol. 4, San Diego, pp. 417-425, 1987.

Pal, S.K. and S. Mitra, Neuro-Fuzzy Pattern Recognition Methods in Soft Computing, John Wiley \& Sons, Inc., New York, 1999. 